\title{
Angle-resolved low coherence interferometry for detection of dysplasia in Barrett's esophagus
}

\author{
Adam Wax ${ }^{1}$, Neil G. Terry ${ }^{1}$, Evan S. Dellon², and Nicholas J. Shaheen ${ }^{2}$ \\ ${ }_{1}^{1}$ Department of Biomedical Engineering, Duke University, Durham, North Carolina \\ ${ }^{2}$ Center for Esophageal Diseases and Swallowing, University of North Carolina, Chapel Hill, North \\ Carolina
}

\section{Keywords}

Barrett's esophagus; optical biopsy; light scattering; dysplasia

\begin{abstract}
Barrett's esophagus (BE) is a metaplastic change of the esophagus which is associated with an increased risk of esophageal adenocarcinoma (EAC) $)^{1}$. The incidence of this cancer has risen dramatically in the U.S. in the last 40 years, ${ }^{2}$ and prognosis for subjects presenting with symptomatic EAC is dismal, with less than a $15 \%$-year survival. ${ }^{3}$ For this reason, strategies aimed at prevention figure prominently in societal guidelines for the care of BE. ${ }^{4}$

Currently, subjects found to have BE on upper endoscopy are often enrolled in endoscopic surveillance programs. In these programs, subjects undergo surveillance endoscopy at intervals governed by the degree of dysplasia found in random biopsies. Subjects developing advanced disease become candidates for intervention, either by endoscopic or surgical means. ${ }^{4}$ While it is intuitive that this approach might avert unresectable EAC, there are several pragmatic issues that threaten the effectiveness of surveillance endoscopy ${ }^{5}$.
\end{abstract}

\section{Dysplasia Detection in BE: A Clinical Conundrum}

A major challenge implicit in our current approaches is the random nature of biopsies taken at surveillance endoscopy. Current guidance suggests that biopsies be taken every $1-2 \mathrm{~cm}$ in each of four quadrants throughout the length of the BE. Even when executed appropriately, this approach samples a scant amount of the surface area, as little as 5\% of the BE. Even more disturbing is recent evidence suggesting that few endoscopists comply with recommendations, and instead take markedly fewer biopsies than suggested per $\mathrm{cm}$ of BE. ${ }^{6}$ Unsurprisingly, multiple studies have suggested significant sampling error in BE

\footnotetext{
(C) 2011 The American Gastroenterological Association. Published by Elsevier Inc. All rights reserved. Corresponding Author: Adam Wax, PhD Box 90281, Durham, NC 27708-0281 A.Wax@ Duke.edu Ph: 919-660-5143 Fax: 919-684-4488.

Author Contributions: AW, NGT, ESD, and NJS were responsible for drafting and editing this manuscript. NGT, AW and NJS were responsible for conducting the literature search. ESD was responsible for creation of the supplemental video.

Disclosures: Adam Wax has a financial interest in Oncoscope, Inc., the company that holds proprietary rights to the a/LCI technology discussed in this review. Neil Terry and Evan S. Dellon have consulting relationships with Oncoscope, Inc. Nicholas J. Shaheen receives research support from Oncoscope, Inc.

Publisher's Disclaimer: This is a PDF file of an unedited manuscript that has been accepted for publication. As a service to our customers we are providing this early version of the manuscript. The manuscript will undergo copyediting, typesetting, and review of the resulting proof before it is published in its final citable form. Please note that during the production process errors may be discovered which could affect the content, and all legal disclaimers that apply to the journal pertain.
} 
surveillance programs, and surgical studies demonstrate that understaging of disease is commonplace. ${ }^{7}$

Misinterpretation of histological specimens also lessens the efficacy of surveillance programs. Given the minute amounts of tissue retrieved, the inherent difficulty is assigning severity of dysplasia, and the confounding effect of any concurrent inflammation, assignment of degrees of dysplasia is fraught with difficulty. This is especially true in the case of low-grade dysplasia, a diagnosis for which agreement between pathologists is at best moderate. ${ }^{8,9}$ Because of variability of pathology reads, confirmation of dysplasia by a second pathologist is recommended. ${ }^{4}$ These confirmatory readings add time and expense

Improvements in endoscopic therapy have further challenged the utility of traditional endoscopic surveillance. In the past, the only available intervention was surgical esophagectomy, and it was largely reserved for subjects with either adenocarcinoma, or as one of several options for high-grade dysplasia. With the increased dissemination of endoscopic ablative therapies, and the accumulating evidence that intervention in the setting of high-grade dysplasia averts EAC, ${ }^{10,11}$ endoscopic intervention is commonly performed in high-grade dysplasia. Some practitioners are opting to perform ablation for lower degrees of dysplasia as well. ${ }^{12}$ The need for histological interpretation of specimens obviates the possibility of therapeutic intervention during the same endoscopic procedure. Instead, a two procedure sequence, whereby the initial endoscopy is diagnostic and involves taking biopsies, and the second is therapeutic, involving ablation or endoscopic mucosal resection of diseased tissue, is performed.

\section{Advanced Endoscopic Imaging in BE}

Given the problems noted above with sampling error, difficulties with interpretation of specimens, and the inability to treat dysplasia at the same endoscopic procedure that it is discovered, there is substantial room for improvement in the care of subjects with BE. One area of great interest is the use of endoscopic imaging techniques. These techniques may either allow identification of areas of increased yield for endoscopic biopsies (so-called "red flag" techniques), or may allow accurate interrogation of smaller areas of BE to assign a degree of dysplasia. The technological challenges facing such modalities are daunting - they must be accurate, easy to apply in the endoscopic setting, and require modest additional training if they seek easy adoption.

A multitude of imaging techniques have been developed, and several have demonstrated promise in cohort studies. The purpose of this review is to assess the current evidence regarding a recently-described imaging modality for $\mathrm{BE}$, angle-resolved low-coherence interferometry (a/LCI $)^{13}$. First, we briefly describe the theory behind a/LCI. Ex and in vivo data regarding the use of the technology to detect dysplasia in the setting of $\mathrm{BE}$ are reviewed. a/LCI is compared to other available and developing technologies for imaging in $\mathrm{BE}$, with respect to degree of resolution, depth of imaging and other characteristics. Finally, we consider likely future developments in BE imaging generally, as well as with respect to a/LCI specifically.

\section{What is a/LCl and How Does it Work?}

The basic premise of a/LCI is that light scattered by cell nuclei produces a pattern that is both discernable and a function of their size and composition. By comparing data retrieved from patient scans to patterns expected for various sized nuclei, a "best fit" is established, allowing an estimate of average nuclear size. In this way, a/LCI takes advantage of a seminal observation in oncogenesis: nuclei undergoing neoplastic degeneration become larger and more pleomorphic on average than nuclei of non-neoplastic cells. Given this 
observation, a technology which accurately measures nuclear size in situ has the potential to detect pre-cancerous change earlier and with greater accuracy than random biopsies. a/LCI imaging has the additional benefit of being "depth-resolved." This means that the device can accurately assess nuclear size at multiple levels in the esophageal wall. As noted below, this depth resolution is vital, since the depth most predictive of the presence of dysplasia corresponds to the basal layer of epithelium. Below, we provide a more detailed description of the a/LCI technique.

To allow assessment of sub-surface nuclei, a/LCI uses a technique called 'coherence gating' to achieve depth resolution, a similar process to that commonly used in optical coherence tomography (OCT). This method allows selective detection of light originating from a specific tissue layer by comparing it with a reference light using an interferometer. a/LCI implements coherence gating by using light wavelengths in the near infrared region of the spectrum. Depth resolution is achieved by having a portion of the light targeted to the tissue while the remainder is used as a reference. Upon combining the scattered light returned from the tissue with the reference light, the resulting interference pattern is detected by wavelength and then processed to provide the depth resolved signal. In a/LCI, the coherence gating process for depth resolution is combined with the high sensitivity of light scattering, allowing measurements of nuclear morphology on a micron scale. Nuclear morphology information is obtained by detecting the scattered light as a function of the angle of the scattered light.

The a/LCI instrumentation consists of an optical engine which provides a light source and receives the scattered light from the tissue, and a fiber probe which delivers and collects light from the tissue (figure 1a) ${ }^{14}$. A specialized fiber probe has been developed which delivers light using a polarization maintaining fiber, as knowledge of the light's polarization is important for enabling accurate light scattering analysis. The fiber probe also collects the light scattered by the tissue using an imaging fiber bundle, with each fiber in the bundle receiving light scattered at a specific angle. The probe incorporates an imaging lens at its tip that is positioned such that each element in the fiber bundle collects light scattered at a particular angle. The fiber probe is 2.3 meters long and approximately 2.5 millimeters wide, enabling application through the accessory channel of most endoscopes (figure 1b), and is enclosed in a plastic sheath. During operation of the a/LCI, the probe is passed through the accessory channel and brought into contact with the epithelium. Data acquisition is initiated by a foot pedal, and typically takes $<40$ milliseconds. During a typical interrogation of a single $1 \mathrm{~mm}^{2}$ site, up to ten measurements are acquired in $<1$ second. There is a raised rim on the tip of the probe, creating a transient tissue indentation that is obvious endoscopically and allows targeting of a concurrent biopsy (figure 1c).

The a/LCI data are analyzed according to a well-documented procedure ${ }^{15}$. In brief, the data are first segmented according to their depth of origin. For each depth segment, the angular distribution of scattered light is analyzed through comparison to Mie theory, an analytical model of scattering by homogeneous spherical objects. The comparison of the actual data to the data expected for scatterers of given sizes allows a "best fit" analysis, yielding an estimate of mean nuclear diameter. This measurement approach has been shown to produce highly accurate nuclear measurements in a variety of cell and tissue types, with a typical accuracy of 0.2-0.3 micrometers. Feasibility studies of a/LCI using animalmodels of neoplasiain the esophagus demonstrate high sensitivity and specificity for discriminating non-dysplastic from dysplastic tissues ${ }^{16}$. A follow on prospective study in the same animal model examined over 80 tissue specimens and produced specificity and sensitivity $>90 \% 17$. 


\section{a/LCI: Clinical Trials}

Recently, a/LCI has been used to assess dysplasia in esophageal tissues. The first application was an examination of ex vivo tissues using a portable instrument ${ }^{18}$. This study examined tissues from just three patients, but showed good sensitivity and specificity when nuclear morphology of dysplastic BE tissue was compared to that of gastric epithelium. While no non-dysplastic BE tissue was encountered in these surgically resected esophageal adenocarcinoma specimens, this study suggested potential for in vivo clinical application.

The first in vivo clinical a/LCI study compared measurements of nuclear morphology from $\mathrm{BE}$ tissues with the histopathological evaluation of co-registered biopsies ${ }^{13}$. The study enrolled 46 patients who were undergoing routine surveillance for BE. During the procedure, the a/LCI fiber probe was applied via the accessory channel to non-nodular BE tissue, and 3-6 sites selected randomly by the endoscopist were scanned. After each of the a/ LCI measurements, the probe was retracted and a co-registered biopsy was taken at the imaging site. Figure $1 \mathrm{~b}$ shows the esophageal mucosa under narrow band imaging (NBI) immediately after application of the probe, and prior to biopsy. In total, 172 co-registered optical and physical biopsies were acquired. The analysis was binary, separating the biopsies as "dysplastic" $(\mathrm{n}=13)$ or "non-dysplastic" $(\mathrm{n}=159)$ according to histopathology. Of available depth-resolved nuclear morphology measurements, the nuclear diameter from the deep epithelium, 200 to $300 \mu \mathrm{m}$ beneath the tissue surface, showed the best discrimination for dysplasia (Figure 1c). The mean nuclear diameter from this layer was highly statistically significant different between dysplastic and non-dysplastic tissues $(\mathrm{P}<0.001)$. Evaluating this metric using a receiver operating characteristic (ROC) curve, very good discrimination was achieved, with an area under the curve (AUC) of 0.91. Based on this analysis, an optimal decision line of nuclear diameter $>11.84 \mu \mathrm{m}$ was determined for classifying dysplasia, yielding a sensitivity of $100 \%$ (13/13), and a specificity of $84 \%(134 / 159)$. The positive and negative predictive values were found to be $34 \%$ (13/38) and 100\% (134/134), respectively. Further trials are underway both to confirm these intriguing results and to continue to improve the diagnostic algorithm.

\section{How Does a/LCl Compare to Other Advanced Imaging Strategies?}

To assess the utility of a/LCI, it is useful to consider previous efforts using optical imaging and spectroscopy methods in BE. A variety of optical techniques have been applied, including autofluorescence, where short wavelength light is absorbed and re-emitted at longer wavelengths, reflectance spectroscopy, which compares the different amounts of light returned at several incident wavelengths, and Raman spectroscopy, which recovers biochemical information by detecting subtle shifts in the frequency of returned light. The strengths and weaknesses of these approaches and others were considered in a recent review ${ }^{19}$. Ultimately, the sensitivity and specificity for detecting dysplasia will be a key metric for utility. Figure 2 summarizes sensitivity and specificity of several recent studies for discriminating normal from diseased BE tissues, including cancer, low grade and high grade dysplasia. Below, we consider the various available advanced imaging technologies with respect to their key operating characteristics: depth of penetration, imaging resolution, need for contrast agents, and ease of use.

The a/LCI technique presents compelling characteristics for discriminating dysplasia in BE because of its depth of penetration. The technique shares a common basis for depth resolution with OCT, which has also been applied in $\mathrm{BE}^{20-23}$. Both technologies offer the ability to probe tissue 1 millimeter, covering the full thickness of the epithelium. Importantly from the clinician's perspective, OCT technologies usually rely on an expert reader to interpret the presence of dysplasia, unlike a/LCI, which reports nuclear diameter directly. To 
address this need, a recent study by Qi et al. ${ }^{22}$, used computer aided diagnosis to retrospectively evaluate OCT images, showing improved sensitivity and specificity.

While most clinical OCT systems can only provide resolution of histological layers, a/LCI is sensitive to sub-cellular structure which can be important for detecting early signs of disease. Specifically, the a/LCI technique uses light scattering to recover information about cell nuclei. Other approaches have shown sensitivity to cell nuclei, including light scattering spectroscopy (LSS) ${ }^{24,25}$ and elastic scattering spectroscopy (ESS) ${ }^{26}$, but these generally lack the ability to obtain depth resolved information. LSS uses polarization to isolate scattering from the surface layer of the epithelium to provide information on nuclear size. ESS on the other hand, uses separated delivery and collection fibers to obtain a signal that arises largely from the lamina propria, although the exact penetration depth can vary with tissue type and probe geometry. The sensitivity of ESS to detect dysplasia is believed to arise from variations in sub-cellular structures such as mitochondria in neoplasia.

Recent advances in endoscopic confocal microscopy have led to its application in assessing dysplasia in BE using a fluorescent contrast agent, typically intravenously-administered fluorescein 27,28 . In this approach, a probe-based confocal microscope is used to image the mucosa, typically to a depth of $100-150 \mu \mathrm{m}$. As with OCT, an expert observer is required to interpret these images. However, the use of a fluorescent contrast agent presents limitations on the duration and repetition of imaging that are not found in label-free techniques.

In addition to the characteristics described above, the clinical ease of use must also be considered when comparing these various imaging modalities. Interpretation of the high resolution data generated by OCT and confocal microscopy may represent a challenge to maintaining patient throughput, and requires training. Like a/LCI, OCT and confocal microscopy also provide detail on only a small area of the mucosa per image. Recent studies have documented efforts for wider-area scanning using $\mathrm{OCT}^{29}$, however, this approach raises new challenges in handling, storage and interpretation of a tremendous volume of data in a time- and cost-conscious manner

To summarize the capabilities of these various modalities, Figure 3 plots their depth penetration and resolution graphically.

\section{Future Directions}

The a/LCI modality and several others discussed here have shown good ability to discriminate dysplastic BE. However, this is not the only consideration for management of BE. Other factors will also greatly influence the adoption of these technologies, including the ease of use, reliability and cost, as well as its impact on the economics of surveillance. Importantly, while several of the technologies have demonstrated proof of principle, data substantiating improved clinically-important outcomes are rare.

It is quite possible that these techniques will be complementary, not competitive. For instance, the ability to couple a wide-field, "red-flag" technique, such as autofluorescence, with a modality that allows interrogation of smaller areas of suspect tissue, such as a/LCI, may provide both the sensitivity and specificity clinicians' desire. Even if these combined modalities solely provided guidance to improve the yield of standard biopsies, such improvement might be worth the time and effort. However, if the accuracy were high enough to obviate the need to perform biopsies, much greater benefits would accrue, including the cost savings of the biopsies and their interpretation, as well as the ability to potentially perform therapeutic intervention at the same endoscopy as the diagnostic examination. Such an approach would be premature based on presently-available data. 
However, given the myriad problems with our current approach, proving superiority of an imaging-based approach to the current standard of care may not be difficult.

Future work with this modality will include validation of the data presented above, and refinement of algorithms for discerning nuclear morphology. Additionally, an intuitive user interface allowing graphic representation of nuclear size with immediate feedback as to the presence of dysplasia and the quality of the signal being attained is necessary. Imaging of larger surface areas of mucosa with a/LCI may be possible and would greatly enhance utility. Finally, epithelial cancer of multiple organs, including colon, lung, and bladder, share many of the same characteristics. Assessment of the potential utility of the device in other organs both in and beyond the GI tract is underway.

\section{Supplementary Material}

Refer to Web version on PubMed Central for supplementary material.

\section{Acknowledgments}

Grant Support: This work was supported in part by the National Institutes of Health (National Cancer Institute R01 CA138594).

Abbreviations
\[ \begin{array}{ll}\text { a/LCI } & \text { angle-resolved low coherence interferometry } \\ \text { AUC } & \text { area under the curve } \\ \text { BE } & \text { Barrett's esophagus } \\ \text { EAC } & \text { esophageal adenocarcinoma } \\ \text { ESS } & \text { elastic scattering spectroscopy } \\ \text { LSS } & \text { light scattering spectroscopy } \\ \text { NBI } & \text { narrow-band imaging } \\ \text { OCT } & \text { optical coherence tomography } \\ \text { ROC } & \text { receiver operating curve }\end{array} \]

\section{References}

1. Shaheen N, Ransohoff DF. Gastroesophageal reflux, barrett esophagus, and esophageal cancer: scientific review. JAMA. 2002; 287:1972-1981. [PubMed: 11960540]

2. Pohl H, Welch HG. The Role of Overdiagnosis and Reclassification in the Marked Increase of Esophageal Adenocarcinoma Incidence. JNCI. 2005; 97:142-146. [PubMed: 15657344]

3. Eloubeidi MA, Mason AC, Desmond RA, et al. Temporal trends (1973-1997) in survival of patients with esophageal adenocarcinoma in the United States: a glimmer of hope. Am J Gastroenterol. 2003; 98:1627-1633. [PubMed: 12873590]

4. Wang KK, Sampliner RE. Updated Guidelines 2008 for the Diagnosis, Surveillance and Therapy of Barrett's Esophagus. Am J Gastroenterol. 2008; 103:788-797. [PubMed: 18341497]

5. Inadomi JM, Sampliner R, Lagergren J, et al. Screening and surveillance for Barrett esophagus in high-risk groups: a cost-utility analysis. Ann Inter Med. 2003; 138:176-186.

6. Abrams JA, Kapel RC, Lindberg GM, et al. Adherence to Biopsy Guidelines for Barrett's Esophagus Surveillance in the Community Setting in the United States. Clin Gastroenterol Hepatol. 2009; 7:736-742. [PubMed: 19268726] 
7. Konda VJA, Ross AS, Ferguson MK, et al. Is the Risk of Concomitant Invasive Esophageal Cancer in High-Grade Dysplasia in Barrett's Esophagus Overestimated? Clin Gastroenterol Hepatol. 2008; 6:159-164. [PubMed: 18096439]

8. Montgomery E, Bronner MP, Goldblum JR, et al. Reproducibility of the diagnosis of dysplasia in Barrett esophagus: A reaffirmation. Hum Pathol. 2001; 32:368-378. [PubMed: 11331953]

9. Alikhan M, Rex D, Khan A, et al. Variable pathologic interpretation of columnar lined esophagus by general pathologists in community practice. Gastrointest Endosc. 1999; 50:23-26. [PubMed: 10385717]

10. Overholt BF, Lightdale CJ, Wang KK, et al. Photodynamic therapy with porfimer sodium for ablation of high-grade dysplasia in Barrett's esophagus: international, partially blinded, randomized phase III trial. Gastrointest Endosc. 2005; 62:488-498. [PubMed: 16185958]

11. Shaheen NJ, Sharma P, Overholt BF, et al. Radiofrequency ablation in Barrett's esophagus with dysplasia. N Engl J Med. 2009; 360:2277-88. [PubMed: 19474425]

12. Lyday D, Corbett W, et al. Radiofrequency ablation of Barrett's esophagus: outcomes of 429 patients from a multicenter community practice registry. Endoscopy. 2010; 42:272-278. [PubMed: 20146164]

13. Terry NG, Zhu Y, Rinehart MT, et al. Detection of Dysplasia in Barrett's Esophagus With In Vivo Depth-Resolved Nuclear Morphology Measurements. Gastroenterology. 2011; 140:42-50. [PubMed: 20854820]

14. Zhu Y, Terry NG, Woosley JT, et al. Design and validation of an angle-resolved low-coherence interferometry fiber probe for in vivo clinical measurements of depth-resolved nuclear morphology. J Biomed Opt. 2011; 16:011003. [PubMed: 21280890]

15. Brown WJWPJ, Terry NG, et al. Review and recent development of angle-resolved low coherence interferometry for detection of pre-cancerous cells in human esophageal epithelium. IEEE J Sel Top Quant. 2008; 14:88-97.

16. Wax A, Yang CH, Muller MG, et al. In situ detection of neoplastic transformation and chemopreventive effects in rat esophagus epithelium using angle-resolved low-coherence interferometry. Cancer Research. 2003; 63:3556-3559. [PubMed: 12839941]

17. Wax A, Pyhtila JW, Graf RN, et al. Prospective grading of neoplastic change in rat esophagus epithelium using angle-resolved low-coherence interferometry. J Biomed Opt. 2005:10.

18. Pyhtila JW, Chalut KJ, Boyer JD, et al. In situ detection of nuclear atypia in Barrett's esophagus using angle-resolved low coherence interferometry. Gastrointest Endosc. 2007; 65:487-491. [PubMed: 17321252]

19. Wilson BC. Detection and treatment of dysplasia in Barrett's esophagus: a pivotal challenge in translating biophotonics from bench to bedside. J Biomed Opt. 2007; 12:051401-22. [PubMed: 17994862]

20. Isenberg G, Sivak MV Jr, Chak A, et al. Accuracy of endoscopic optical coherence tomography in the detection of dysplasia in Barrett's esophagus: a prospective, double-blinded study. Gastrointest Endosc. 2005; 62:825-31. [PubMed: 16301020]

21. Evans JA, Poneros JM, Bouma BE, et al. Optical coherence tomography to identify intramucosal carcinoma and high-grade dysplasia in Barrett's esophagus. Clin Gastroenterol Hepatol. 2006; 4:38-43. [PubMed: 16431303]

22. Qi X, Pan Y, Sivak MV, et al. Image analysis for classification of dysplasia in Barrett's esophagus using endoscopic optical coherence tomography. Biomed Opt Express. 2010; 1:825-847. [PubMed: 21258512]

23. Zagaynova E, Gladkova N, Shakhova N, et al. Endoscopic OCT with forward-looking probe: clinical studies in urology and gastroenterology. J Biophotonics. 2008; 1:114-28. [PubMed: 19343643]

24. Wallace MB, Perelman LT, Backman V, et al. Endoscopic detection of dysplasia in patients with Barrett's esophagus using light-scattering spectroscopy. Gastroenterology. 2000; 119:677-682. [PubMed: 10982761]

25. Georgakoudi I, Feld MS. The combined use of fluorescence, reflectance, and light-scattering spectroscopy for evaluating dysplasia in Barrett's esophagus. Gastrointest Endosc Clin N Am. 2004; 14:519-537. [PubMed: 15261200] 
26. Lovat LB, Johnson K, Mackenzie GD, et al. Elastic scattering spectroscopy accurately detects high grade dysplasia and cancer in Barrett's oesophagus. Gut. 2006; 55:1078-83. [PubMed: 16469795]

27. Pohl H, Rosch T, Vieth M, et al. Miniprobe confocal laser microscopy for the detection of invisible neoplasia in patients with Barrett's oesophagus. Gut. 2008; 57:1648-53. [PubMed: 18755886]

28. Kiesslich R, Gossner L, Goetz M, et al. In Vivo Histology of Barrett's Esophagus and Associated Neoplasia by Confocal Laser Endomicroscopy. Clin Gastroenterol Hepatol. 2006; 4:979-987. [PubMed: 16843068]

29. Suter MJ, Vakoc BJ, Yachimski PS, et al. Comprehensive microscopy of the esophagus in human patients with optical frequency domain imaging. Gastrointest Endosc. 2008; 68:745-753. [PubMed: 18926183] 


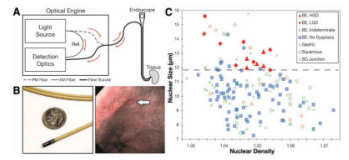

Figure 1.

A) Simplified a/LCI system diagram. Red arrows indicate direction of light: delivery of light using polarization maintaining (PM) fiber, collection of angular scattering using a fiber bundle and the reference arm using single mode (SM) fiber. B) Images of a/LCI probe tip next to a US dime for scale (left) and the mark left by the probe (right, arrow). C) a/LCI in vivo clinical trial results. Points are colored according to their pathological diagnosis and plotted as a function of their nuclear size and density as measured by a/LCI. Dashed line indicates an optimal decision line identifying dysplastic tissue sites with a sensitivity of $100 \%(13 / 13)$ and a specificity of $84 \%$ (134/159). Adapted from Reference ${ }^{13}$. 


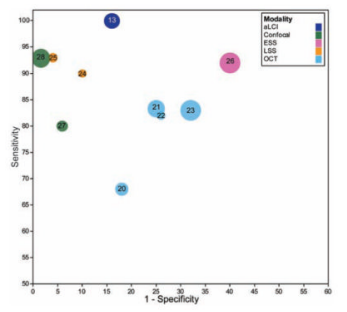

Figure 2.

Sensitivity and specificity relationship between in vivo BE dysplasia studies, plotted on an ROC curve. Numbers indicated correspond to bibliographic reference number. This plot shows the ability of the studies to differentiate LGD+HGD+Cancer from healthy tissue when data are available. In the case of only HGD+ differentiation being performed, that figure is used instead. Circle size indicates the number of patients in the study. 


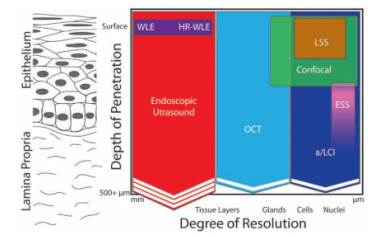

Figure 3.

Qualitative comparison of resolution/depth penetration for various endoscopic advanced imaging modalities. The $\mathrm{Y}$ axis of the figure denotes the depth to which the imaging modality can probe the tissue. The $\mathrm{X}$ axis denotes the degree of resolution, with modalities on the right side of the figure providing a higher degree of resolution than those on the left. Chevrons indicate the ability of a modality to penetrate deeper than indicated and increased numbers of chevrons indicates increased penetration ability. 